\title{
Injury Risk to Extraosseous Knee Vasculature During Osteotomies: A Cadaveric Study With CT and Dissection Analysis
}

\author{
Salvatore Bisicchia MD, Federica Rosso MD, \\ Marc A. Pizzimenti PhD, Chamnanni Rungprai MD, \\ Jessica E. Goetz PhD, Annunziato Amendola MD
}

Received: 7 May 2014/ Accepted: 7 October 2014/Published online: 22 October 2014

(C) The Association of Bone and Joint Surgeons(B 2014

\begin{abstract}
Background Realignment osteotomies about the knee may be performed as distal femoral or proximal tibial osteotomies; both may be performed either on the medial or lateral sides of the knee, in closing- or opening-wedge fashion. Although rare, injury to neurovascular structures may occur, and the proximity of the vascular structures to the osteotomy saw cuts has been incompletely characterized.

Questions/purposes We performed a cadaver study to assess the risk of vascular injury in patients undergoing realignment osteotomies by (1) quantifying the distances between osteotomy saw cuts and blood vessels using three-
\end{abstract}

One of the authors certifies that he (SB) has received grants during the study period, an amount of USD 10,000 to 100,000 from "Fondazione Enrico ed Enrica Sovena" (Rome, Italy), a nonprofit foundation that partially sponsored his fellowship at the Department of Orthopaedics and Rehabilitation of the University of Iowa.

All ICMJE Conflict of Interest Forms for authors and Clinical Orthopaedics and Related Research editors and board members are on file with the publication and can be viewed on request.

Ethical committee approval was waived for this work at University of Iowa Hospitals and Clinics.

The work was performed at Department of Orthopaedics and Rehabilitation, University of Iowa Hospitals and Clinics, Iowa City, IA, USA.

\section{S. Bisicchia $(\square)$}

Department of Orthopaedic Surgery, University of Rome Tor Vergata, Viale Oxford 81, 00133 Rome, Italy

e-mail: s.bisicchia@gmail.com

F. Rosso

Department of Orthopedics and Traumatology, AO Mauriziano Umberto I, Turin, Italy

\section{A. Pizzimenti}

Department of Anatomy and Cell Biology, University of Iowa, Iowa City, IA, USA dimensional CT reconstruction after distal femoral and proximal tibial osteotomies; and (2) qualitatively describing the small- and medium-sized vasculature around the knee, to provide the link between the CT analysis and wedge incision measures, and better show the potential extraosseous supply to the regions investigated.

Methods Twelve human cadaveric knees were injected with a latex and barium sulfate suspension into the superficial femoral artery. Each specimen underwent CT to evaluate vascular perfusion and was randomized to either a lateral opening-wedge distal femoral osteotomy and medial opening-wedge proximal tibial osteotomy group, or a medial closing-wedge distal femoral osteotomy and lateral closing-wedge proximal tibial osteotomy group. Postoperatively, knees underwent $\mathrm{CT}$ in extension to measure the shortest distance between the osteotomies and the popliteal artery, anterior and posterior tibial arteries, and genicular arteries. Vessels between $5 \mathrm{~mm}$ and $10 \mathrm{~mm}$ from the osteotomy cut were considered in a zone of moderate risk for damage, while vessels less than $5 \mathrm{~mm}$ from the cut were considered in a zone of high risk for damage. Vessels more than $10 \mathrm{~mm}$ from the cut were not considered to be at risk. Subsequently, knees underwent dissection and chemical débridement to qualitatively describe the smaller

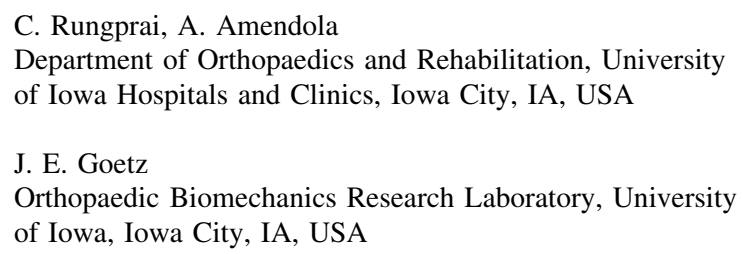


vessels. This part of the study added visual information and gave a comprehensive overview of the vessels at risk.

Results All variations of the osteotomies put at least one artery at risk. The popliteal artery was found in a risk zone for injury in two specimens during closing-wedge distal femoral osteotomy (median distance, $11.6 \mathrm{~mm}$; range, 5.2-14.6 mm). The superior lateral genicular artery was in a risk zone in all the specimens during opening-wedge distal femoral osteotomy (median distance, $3.0 \mathrm{~mm}$; range, $0.7-6.5 \mathrm{~mm}$ ), and in five specimens during closing-wedge distal femoral osteotomy (median distance, $4.5 \mathrm{~mm}$; range, $1.3-11.2 \mathrm{~mm}$ ). A concomitant risk for superior medial genicular artery injury was observed in five specimens during opening-wedge distal femoral osteotomy (median distance, $8.7 \mathrm{~mm}$; range, 0.8-13.9 mm) and in four specimens during closing-wedge distal femoral osteotomy (median distance, 4.1 ; range, $0.5-41.7 \mathrm{~mm}$ ). The popliteal artery was in a risk zone in four specimens during openingwedge proximal tibial osteotomy (median distance, $9.6 \mathrm{~mm}$; range, 6.6-12.9 $\mathrm{mm}$ ), and in three specimens during closing wedge proximal tibial osteotomy (median distance, $9.6 \mathrm{~mm}$; range, $4.4-11 \mathrm{~mm}$ ). The inferior medial genicular artery could be classified at risk in five specimens during opening-wedge proximal tibial osteotomy (median distance, $2.1 \mathrm{~mm}$; range, 0.3-32 mm) and in five specimens during closing-wedge proximal tibial osteotomy (median distance, $5.8 \mathrm{~mm}$; range, 1.4-13 mm). Furthermore, the inferior lateral genicular artery was found in a risk zone in two specimens of closing-wedge proximal tibial osteotomies (median distance, $17.4 \mathrm{~mm}$; range, 8-23.3 mm). There were no differences between openingwedge and closing-wedge distal femoral osteotomies and proximal tibial osteotomies in the vessels at risk during the procedure. After chemical débridement, knees showed abundant vascularization of the distal femur and lateral tibia, whereas the medial tibia contained few arteries.

Conclusions With the numbers available, we found that none of the osteotomy techniques performed was safer than any other in terms of the proximity of the major arterial structures and some vessels appear to be at relatively high risk during these procedures.

Clinical Relevance This study clarifies that the genicular arteries on the opposite side of the surgical field, which cannot be seen and protected during the procedure, can be at risk of injury, particularly when the cortical hinge is compromised. Additional studies are necessary to address the potential risk of the dissection needed for plate placement and injuries related to drilling and screw placement during osteotomies around the knee.

\section{Introduction}

Osteoarthritis of the knee is common and may be associated with either varus or valgus lower-limb malalignment.
Contributing factors, including trauma or previous surgeries, such as total meniscectomy or ACL reconstruction, also may lead to early osteoarthritis associated with malalignment in young, active patients [8, 23]. For younger patients, a realignment osteotomy may be performed as a distal femoral or proximal tibial osteotomy, or both, depending on the deformity of the lower limb. Distal femoral and proximal tibial osteotomies have been performed on either the medial or lateral side, with satisfactory clinical postoperative results [32, 36, 38]. In most cases, a medial opening-wedge proximal tibial osteotomy (in a varus knee), or a lateral opening-wedge distal femoral osteotomy (in a valgus knee) is indicated, but other options, such as a lateral closing-wedge proximal tibial osteotomy or medial closing-wedge distal femoral osteotomy may be appropriate in individual cases since each type of osteotomy has its specific advantages and disadvantages. Osteotomies also can be performed as associated procedures in patients undergoing cartilaginous surgery [4], meniscal allograft transplantation [2], or ligament reconstruction [26]. In such cases, mechanical realignment may have a protective effect on the reconstructed or transplanted structures without significantly increasing complication rates [39].

Considering all the possible osteotomies about the knee, the lateral closing-wedge proximal tibial osteotomy has the highest complication rate [35]. The most severe, albeit rare, complication is damage to the arteries and nerves about the knee [9, 14, 27-29, 33]. Delayed union and nonunion also can complicate osteotomies around the knee [35]. Although there may be several predisposing factors related to poor healing of the osteotomy, damage to small- and mediumsized vessels during the procedure may be important to consider.

We therefore sought to assess the risk of vascular injury in osteotomies around the knee by (1) quantifying for the first time using three-dimensional (3-D) reconstructions the distances between osteotomy saw cuts and blood vessels after distal femoral and proximal tibial osteotomies in openingwedge and closing-wedge procedures; and (2) qualitatively describing the small- and medium-sized vasculature around the knee to provide visual confirmation of neighboring structures in the surgical field and outline a comprehensive overview of the vessels at risk. Addressing these issues should add to understanding of the vascularity of the knee in the context of osteotomies.

\section{Materials and Methods}

A pilot study was completed with two specimens to test the feasibility of assessing vascular damage, the possibility of performing two osteotomies in the same knee without 
substantial modification of the vascular anatomy, and the timing for chemical débridement. Without additional anatomic dissection, chemical débridement was incomplete in the first specimen even after 12 hours, a much longer time than expected from previous studies [21, 34], likely related to the more substantial soft tissue envelope around the knee compared with the foot and ankle. Therefore, more complete anatomic dissection was introduced in the study protocol and successfully tested on the second specimen to obtain complete débridement.

For our main study, 12 frozen human cadaveric knees (Anatomy Gifts Registry, Baltimore, MD, USA), with a mean cadaver age of 71.4 years (range, 65-84 years) were thawed overnight at room temperature. Specimens with evidence of prior knee trauma, surgery, or deformity were excluded. Specimens were imaged under an image intensifier to exclude cases of significant varus or valgus deformity or severe osteoarthritis. History of knee injury or prior knee pain was unknown for all specimens. The superficial femoral artery was identified and cannulated with an 18-gauge cannula, with the cannula sutured in place to occlude the proximal end of the artery. The artery was injected with normal saline until the effluent from the anterior tibial artery, posterior tibial artery, and peroneal artery was clear. All three effluent arteries were clamped to improve perfusion of all vessels and the major vessel was injected with a 1:2 suspension of barium sulfate powder to water, mixed with Ward's Blue Latex (Ward's Science, Rochester, NY, USA) (1 part suspension: 2 parts latex) through the cannula, as previously described [21, 34].

The same day, the knees underwent CT to evaluate vascular perfusion with a SOMATOM ${ }^{\circledR}$ Definition Flash scanner (Siemens AG, Munich, Germany) to determine if perfusion with barium provided adequate observation of the vessels. The evaluation protocol used was CT acquisition reference mAs/actual $250 \mathrm{mAs}, 120 \mathrm{kV}$, exposure time 0.5 second, reconstruction thickness $0.6 \mathrm{~mm}$, reconstruction spacing $0.4 \mathrm{~mm}$, and display field of view $170 \mathrm{~mm}$. Perfusion was deemed adequate in all knees.

After the CT scans, the knees were labeled and kept in separate marked plastic bags for future identification, frozen again, and randomized to either one of two groups (two procedures in each knee; six knees in each group): (1) opening-wedge group: a lateral opening-wedge distal femoral osteotomy and a medial opening-wedge proximal tibial osteotomy were performed; or (2) closing-wedge group: a medial closing-wedge distal femoral osteotomy and a lateral closing-wedge proximal tibial osteotomy were performed.

\section{Surgical Techniques}

The knees were thawed overnight to room temperature. Commercially available surgical instrumentation was used, but no actual implants were placed. Tibial osteotomy was performed first in each specimen. In every case, a 10-mm wedge either was opened or closed since this is a common amount of correction required in clinical practice [10]. In the opening-wedge group (on the femur and tibia), the osteotomy gap was kept open by a $10-\mathrm{mm}$ bone wedge taken from the femoral or tibial shaft.

\section{The Opening-wedge Proximal Tibial Osteotomy}

An anteromedial vertical skin incision was made midway between the medial border of the tibial tubercle and the posterior border of the tibia, just anterior to the pes anserine and $1 \mathrm{~cm}$ below the joint line. The sartorial fascia was incised just medial to the patellar tendon, which was elevated slightly, to observe the hamstrings and superficial medial collateral ligament. A longitudinal incision was made through the periosteum, and the periosteum was elevated off the tibia along with the hamstrings and superficial fibers of the medial collateral ligament. A blunt retractor was placed behind the back of the tibia without any soft tissue interposition to protect the neurovascular structures, and another retractor was positioned to protect the patellar tendon. Under fluoroscopic control, a guide wire was positioned from medial to lateral, at the level of the superior aspect of the tibial tubercle, arriving $1 \mathrm{~cm}$ below the joint line and starting from $4 \mathrm{~cm}$ below the medial joint line, careful to stay above the tibial tuberosity. A cortical osteotomy was performed using an oscillating saw, positioned inferiorly to the guide wire to prevent superior migration of the cut and intraarticular fracture. The osteotomy cut was extended with an osteotome under fluoroscopic control. Once the osteotomy was completed, the medial opening was created using progressively larger osteotomes placed at least $1 \mathrm{~cm}$ from the lateral cortex, and a dedicated triangle osteotomy wedge (osteotomy wedge trials, Arthrex, Naples, FL, USA) positioned to the desired depth. The osteotomy gap was kept open with a 10-mm wedge taken from the tibial diaphysis.

\section{The Opening-wedge Distal Femoral Osteotomy}

An incision was made through the skin and subcutaneous tissue, starting from the lateral femoral epicondyle and directed proximally for approximately $6 \mathrm{~cm}$. The iliotibial band was incised and the vastus lateralis was elevated off the lateral intermuscular septum. Blunt retractors were inserted anteriorly and posteriorly around the femur. The periosteum was elevated off the femur. Under fluoroscopic control, a guide pin was inserted proximally and laterally (from the metadiaphyseal junction) and directed distally 
and medially, just to the corner of the medial epicondyle. An oscillating saw was used to cut through the lateral cortex and then progressively larger osteotomes were placed at least $1 \mathrm{~cm}$ from the medial cortex. A dedicated triangle osteotomy wedge was positioned to the desired depth. The osteotomy gap was kept open with a $10-\mathrm{mm}$ wedge taken from the femoral diaphysis.

\section{The Closing-wedge Proximal Tibial Osteotomy}

For the closing-wedge proximal tibial osteotomy, a curved skin incision was made starting posteriorly at the lateral joint line and directed distally midway between the tibia and the fibula. The fascia was incised just distal to Gerdy's tubercle. The tibialis anterior muscle was detached and elevated off the proximal tibia and retracted laterally, exposing the proximal tibiofibular joint. With a scalpel blade, a slice of the articular cartilage of the fibula was cut to dislocate the joint. Tissues were incised just lateral to the patellar tendon, which was elevated slightly. The periosteum was elevated off the tibia and a blunt retractor was inserted posteriorly around the bone. Under fluoroscopic control, a guide pin was placed $1.5 \mathrm{~cm}$ distal and parallel to the joint line above the tibial tubercle. An oscillating saw was used to cut through the lateral cortex. A second guide wire was placed $10 \mathrm{~mm}$ distally, directed toward the tip of the first guide wire, and the cortex was cut with an oscillating saw. Osteotomes and rongeurs were used to complete the cut medially and remove a $10-\mathrm{mm}$ bone wedge. A valgus stress was applied to the knee to close the osteotomy and \#2 FiberWire ${ }^{\circledR}$ transosseous sutures (Arthrex) were used to secure closure to avoid interference during postoperative $\mathrm{CT}$ scanning.

\section{The Closing-wedge Distal Femoral Osteotomy}

An incision through skin and subcutaneous tissues was made on the medial aspect of the femur starting from the medial epicondyle. The fascia was incised and elevated. The vastus medialis was elevated off the intermuscular septum. Two blunt retractors were placed on the anterior and posterior aspects of the femur. The periosteum was elevated off the femur and a guide wire placed at the level of the medial epicondyle parallel to the joint line. A chisel was placed proximal to the guide wire to reproduce the blade plate used in clinical practice at our institution. Under fluoroscopic control, a guide wire was placed medial to lateral, at the level of the metadiaphyseal junction, parallel to the joint line. A 10-mm wedge was removed proximal to the guide wire using an oscillating saw, osteotomes, and rongeurs. Varus stress was applied to the

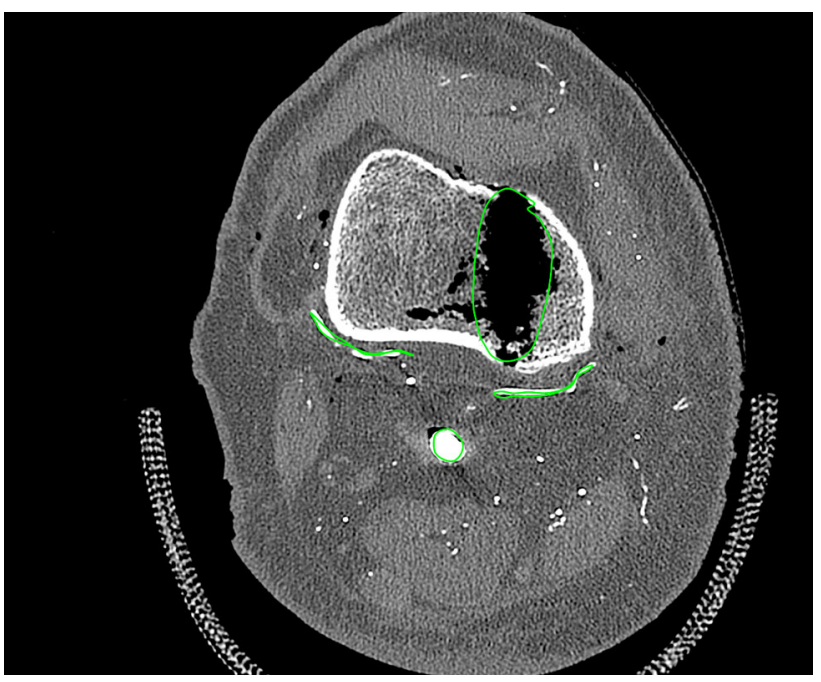

Fig. 1 The studied vessels were traced manually on a touch screen personal computer, using postoperative CT scans to measure the shortest distance to the osteotomy cuts on a three-dimensional model.

knee to close the osteotomy and \#2 FiberWire ${ }^{\circledR}$ transosseous sutures were used to secure closure.

\section{CT Analysis}

After surgical completion, the knees underwent postoperative CT with the same protocol previously described. Postoperative CT scans were analyzed using image-viewing software (OsiriX; Pixmeo, Bernex, Switzerland) on a personal computer with a touch screen. A region of interest was placed manually with a stylus on the touch screen to contour the popliteal, anterior tibial, posterior tibial, superior lateral genicular, superior medial genicular, inferior lateral genicular, and inferior medial genicular arteries, and the osteotomies (Fig. 1). Segmented data were exported for analysis using a custom routine developed in MATLAB ${ }^{\circledR}$ (Mathworks, Natick, MA, USA) to determine the shortest Euclidean distance between each vessel and the osteotomy cuts using the 3-D model. These data were used to create a "risk map." Vessels between $5 \mathrm{~mm}$ and $10 \mathrm{~mm}$ from the osteotomy cut were considered in a zone of moderate risk for damage, while vessels less than $5 \mathrm{~mm}$ from the cut were considered in a zone of high risk for damage. Vessels more than $10 \mathrm{~mm}$ from the cut were not considered to be at risk.

\section{Chemical Débridement and Anatomic Dissection}

After postoperative CT data were analyzed, specimens were thawed overnight and prepared for dissection. Skin and subcutaneous tissues were sharply dissected away. All 
tissue located $4 \mathrm{~cm}$ or more proximal to the femoral osteotomy and $3 \mathrm{~cm}$ or more distal to the tibial osteotomy were removed, leaving only bone. Based on our pilot study, partial removal of muscle mass significantly reduced timeconsuming chemical débridement; however, the vessels which were measured during the $\mathrm{CT}$ scan and their nearby surrounding soft tissues were left intact to prevent iatrogenic injury to these vessels. The rectus femoris, vastus lateralis, biceps femoris, medial and lateral gastrocnemius, sartorius, gracilis, semitendinosus, and semimembranosus muscles were carefully debulked and detached from their origins and/or insertions to expose the popliteal fossa.

After gross dissection, specimens were submerged in $6.0 \%$ sodium hypochlorite for 4 hours to complete débridement of soft tissues. The specimens were checked every 30 minutes and the sodium hypochlorite was refreshed as needed. Débridement was stopped once overlying soft tissues had been débrided adequately to allow examination of the vessels surrounding the osteotomy cuts (although the vessel walls were débrided with the sodium hypochlorite, the blue latex casts of the vessel remained). Further detailed dissection was performed to expose the vessels of interest. Vessels were examined carefully and photographed to document injury or lack of injury (Fig. 2). Chemical débridement and anatomic dissection were used for direct observation and qualitative assessment of proximity between vessels and osteotomies. No physical measurements were made on the débrided specimens in case the absolute tissue relationships had been slightly distorted by the surgical or chemical débridement of soft tissues.

All surgical procedures and débridements were performed by the same author (SB). The study was exempt from institutional review board approval at the University of Iowa.

\section{Statistical Methods}

Descriptive statistics were used to report the distances between the studied vessels and the osteotomy cuts. Measurements have been reported in millimeters (rounded to the first decimal); medians and ranges were provided as needed. Statistical analysis was not performed owing to the limited number of specimens included in this study.

\section{Results}

Proximity of Arterial Structures to Osteotomy Sites

In each of the four osteotomies studied, at least one artery was in a zone of moderate or high risk of injury owing to its
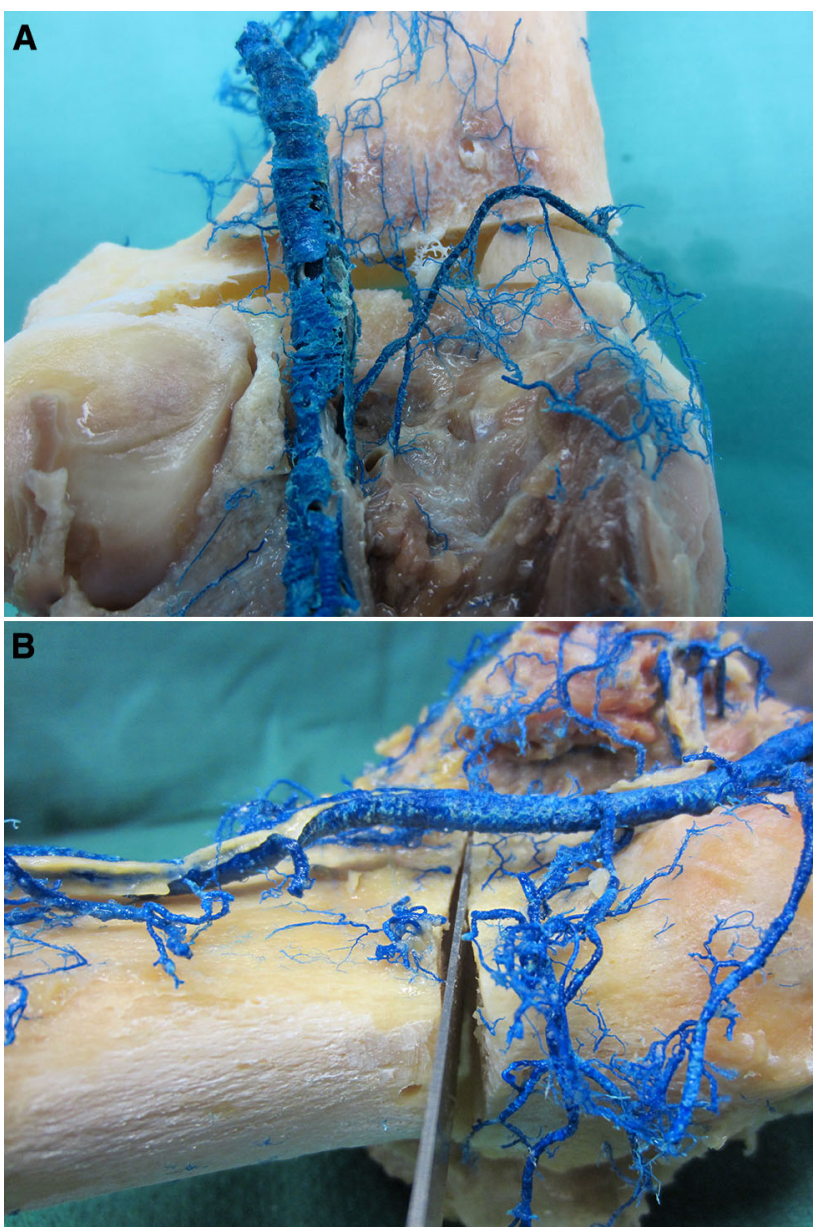

Fig. 2A-B (A) After anatomic dissection and chemical débridement, the cadaveric specimens were photographed. The proximity of the superior lateral genicular artery to the osteotomy line in a specimen that underwent opening-wedge lateral distal femoral osteotomy is evident. (B) In this specimen, the proximity of the popliteal artery during opening-wedge medial proximal tibial osteotomy is seen. In this latter specimen, branches of the inferior medial genicular artery were cut during surgery.

proximity to the osteotomy site (Table 1). The popliteal artery was found in a zone of moderate risk for injury in two specimens during closing-wedge distal femoral osteotomy (median distance, $11.6 \mathrm{~mm}$; range, $5.2-14.6 \mathrm{~mm}$ ). The superior lateral genicular artery was in a risk zone in all the specimens (five high, one moderate) during opening-wedge distal femoral osteotomy (median distance, $3.0 \mathrm{~mm}$; range, $0.7-6.5 \mathrm{~mm}$ ), and in five specimens (three high, two moderate) during closing-wedge distal femoral osteotomy (median distance, $4.5 \mathrm{~mm}$; range, $1.3-11.2 \mathrm{~mm}$ ). A concomitant risk for superior medial genicular artery injury was observed in five specimens (one high, four moderate) during opening-wedge distal femoral osteotomy (median distance, $8.7 \mathrm{~mm}$; range, $0.8-13.9 \mathrm{~mm}$ ) and in four specimens (three high, one moderate) during closing-wedge distal femoral osteotomy (median distance, $4.1 \mathrm{~mm}$; range, $0.5-41.7 \mathrm{~mm}$ ). 


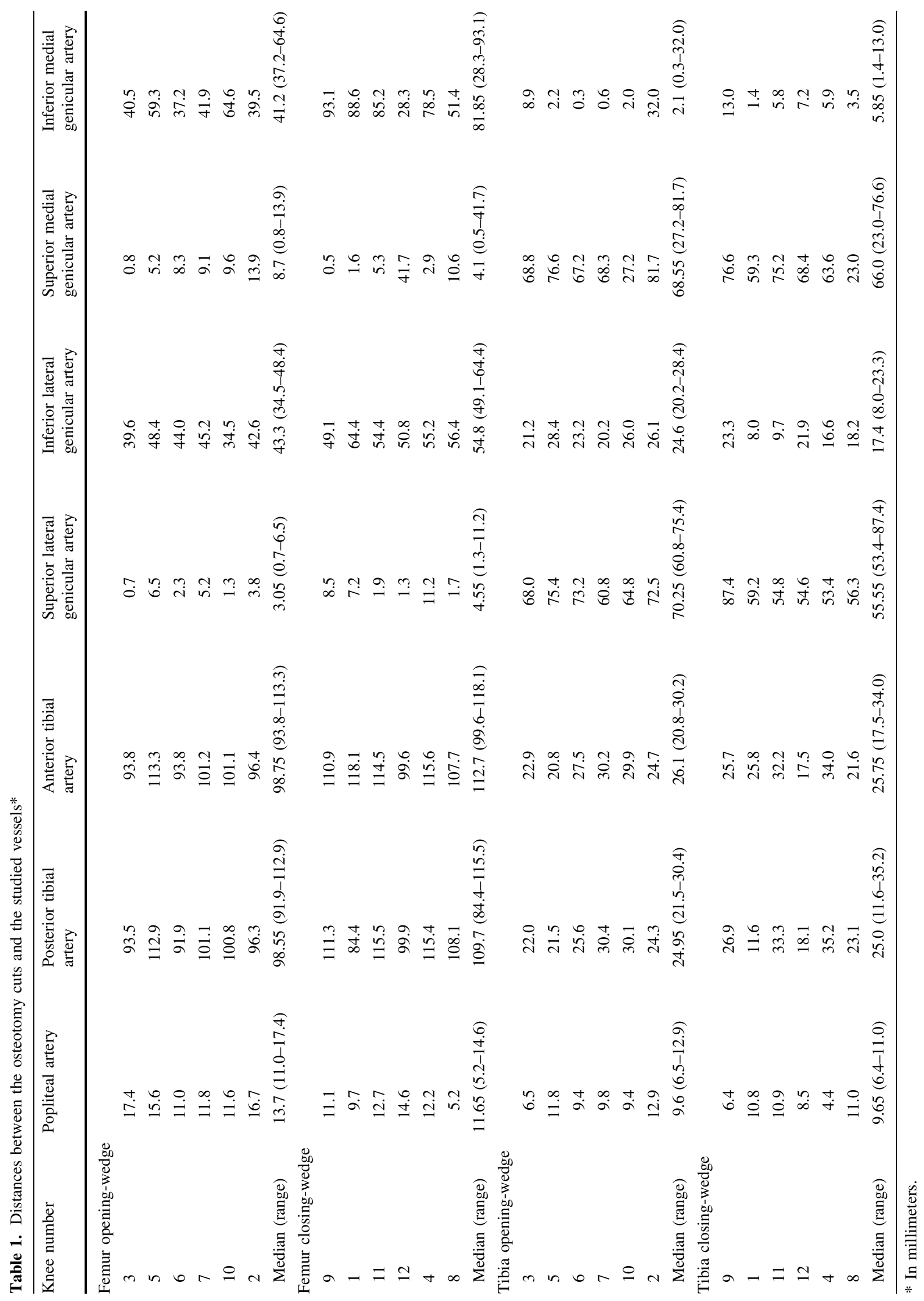


The popliteal artery was in a zone of moderate risk for injury in four specimens during opening-wedge proximal tibial osteotomy (median distance, $9.6 \mathrm{~mm}$; range, 6.5$12.9 \mathrm{~mm}$ ), and in three specimens (one high, two moderate) during closing-wedge proximal tibial osteotomy (median distance, $9.6 \mathrm{~mm}$; range, $6.4-11 \mathrm{~mm}$ ). Furthermore, given its vascular path, the inferior medial genicular artery could be classified at risk in five specimens (four high, one moderate) during opening-wedge proximal tibial osteotomy (median distance, $2.1 \mathrm{~mm}$; range, $0.3-32 \mathrm{~mm}$ ) and in five specimens (two high, three moderate) during closing-wedge proximal tibial osteotomy (median distance, $5.8 \mathrm{~mm}$; range, $1.4-13 \mathrm{~mm}$ )., The inferior lateral genicular artery was found in a zone of moderate risk in two specimens during closing-wedge proximal tibial osteotomy (median distance, $17.4 \mathrm{~mm}$; range, $8-23.3 \mathrm{~mm}$ ).

\section{Qualitative Analysis of Smaller Vessels}

Qualitative analysis of the debrided knees showed abundant vascularization on both sides of the femur and in the lateral compartment of the leg, while the medial side of the tibia was supplied mainly by subcutaneous vessels and showed few arteries (Fig. 3).

One of the knees (Specimen 1) had an anatomic variant, with the peroneal artery originating very proximal from the popliteal artery (together with the posterior tibial artery), and the anterior tibial artery originating very distally from the peroneal artery (Fig. 4). In Specimen 1, the distance between the peroneal artery and closing-wedge proximal tibial osteotomy was $8.5 \mathrm{~mm}$ and the vessel was considered in a moderate-risk zone for damage.

\section{Discussion}

Damage to the surrounding neurovascular structures is a substantial operative complication of osteotomies around the knee [9, 14, 27-29, 33]. Previous studies have focused primarily on associated risk to the popliteal artery and how to prevent its injury during proximal tibial osteotomy [9, $14,27-29,33]$, but there is a paucity of literature regarding vascular risks related to distal femoral osteotomy [37]. We therefore sought to better quantify the risk of damage to major blood vessels from osteotomies performed around the knee and to assess qualitatively the small- and mediumsized vasculature.

Limitations exist in our study. The small sample size of each group may have led to a type II error and inability to detect a difference between the opening-wedge and closing-wedge groups. We did not use entire lower extremities from the hip to the foot as described by Kim et al. [14]. The
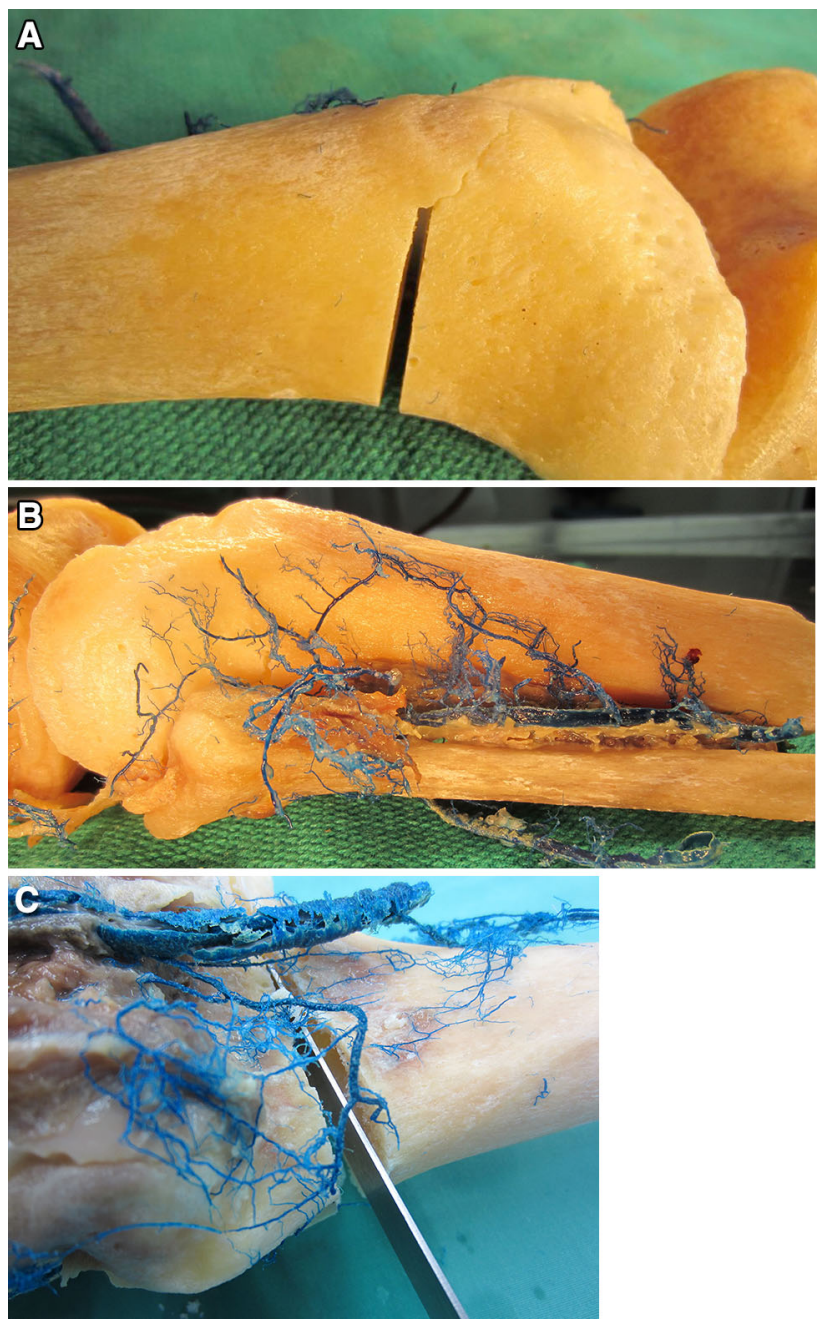

Fig. 3A-C The (A) medial side of the proximal tibia is mainly subcutaneous with an obvious lack of vessels compared with the (B) lateral side of the proximal tibia or the $(\mathbf{C})$ lateral side of the femur.

average age of the donors of specimens in our study was 71.4 years, substantially older than patients usually undergoing osteotomies around the knee [10, 32]. Even if we excluded severely arthritic knees, the age of the specimens may have led to the inclusion of knees with lowgrade osteoarthritis, which may have affected the results of our study because the relationship between the proximal tibia and popliteal artery can differ in arthritic knees [7]; however, this difference is reported to be minimal $(1 \mathrm{~mm})$ and its clinical implications questionable [16]. Therefore, the age of the donors and the inclusion of knees with lowgrade osteoarthritis did not bias the results of our study. Two osteotomies were performed in each knee to reduce the number of specimens needed, but this did not substantially alter the vascular anatomy of the lower limb. Although all osteotomies were performed to replicate with high fidelity the actual surgical procedures, internal fixation devices were not used and some damage to vascular 


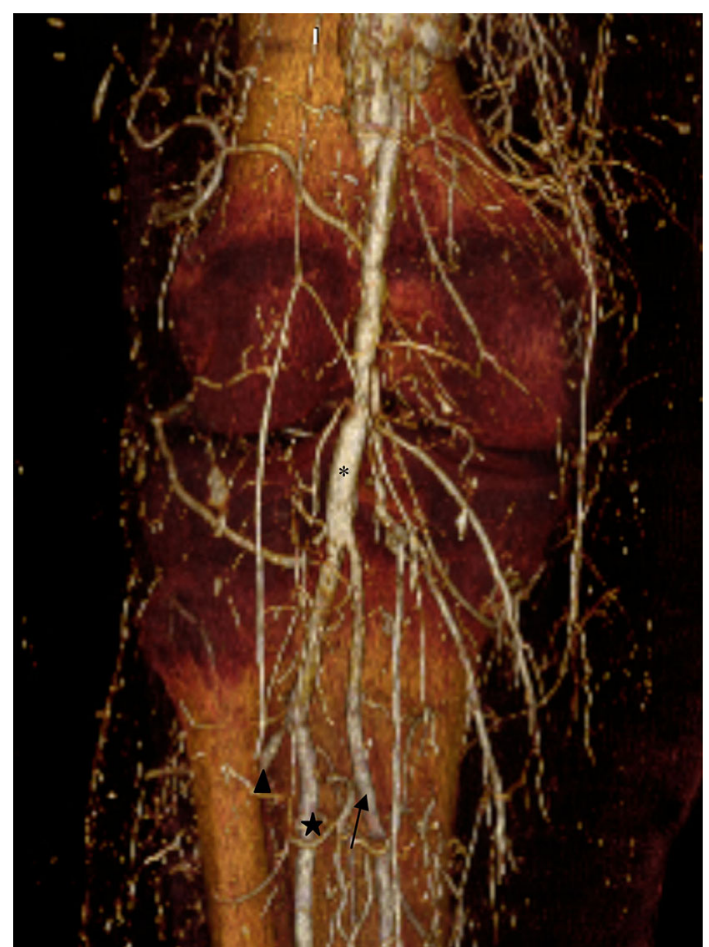

Fig. 4 One of the knees had an anatomic variant, with the peroneal artery (arrow) originating very proximally from the popliteal artery (*), together with the posterior tibial artery (star), and the anterior tibial artery (arrow head) originating very distally from the peroneal artery. The distance between the peroneal artery and closing-wedge proximal tibial osteotomy was $8.5 \mathrm{~mm}$ and the peroneal artery was considered to be in a moderate risk zone for damage.

structures in these regions in actual patients may be the result of drill or screw penetration. We chose not to position and internally fix plates, since the metal would result in CT scatter. In the previous cadaveric studies, Visser et al. [37] used a plate and screws in the only specimen in which they performed their minimally invasive medial distal femoral osteotomy, however, Kim et al. [14] did not use any internal fixation to determine vascular injuries during proximal tibial osteotomy. Within-group variability for vessel measures and the osteotomy cuts may be related to an underpowered sample size, anatomic variability between individuals, or both. Complete study of the angiosome distribution was not part of this investigation. The angiosomes of the genicular region are vast with supply not only from popliteal branching but also from the descending branch of the lateral femoral circumflex artery. Although these contributions may be important to consider for the superficial structures associated with distal femoral and proximal tibial osteotomies, our study did not detail these contributions. One study quantifying the potential anastomotic details associated on the lateral knee indicated that these connections may not be as robust, common, or as functionally available as thought, particularly in the surgical context [24]. These limitations are balanced by the strengths of our study: (1) distances between osteotomy cuts and vessels were not measured at preestablished points, but for the first time on a 3-D model on CT scan sections, allowing for determination of the shortest possible distance; (2) all the different types of osteotomies around the knee were performed; and (3) anatomic dissection allowed for better observation of the relationship between osteotomies and vessels.

In our study, for all approaches, the proximity of vessels to the osteotomy site posed risk of injury to at least one major artery (Table 1). We draw particular attention to the genicular arteries, especially those on the opposite side of the surgical field that cannot be seen and protected during the procedure, since they are at risk of injury when the cortical hinge is compromised. Given the limited plasticity of cortical bone, such events are not rare and have been reported to occur in as much as $90 \%$ of cases in which an angular correction greater than $8^{\circ}$ is required [3]. All approaches seem to have risk, although the risks differ by approach; none seems completely safe, and our study was too small to determine whether one is safer than another. In our study, the superficial medial collateral ligament was detached and reflected to place a retractor behind the tibia. Getgood et al. [10] reported similar management of this ligament, while Kim et al. [14] did not detail this aspect of their surgical approach. Similar to our study, screws, plates, or drilled screw holes were not used in those studies $[10,14]$. To prevent lateral cortex breakthrough, we recommend using a short $(50 \mathrm{~mm})$ oscillating saw to cut the cortex, and then complete the osteotomy with osteotomes and rongeurs under image intensification. More studies are needed to address the potential risk added by the dissection needed for plate placement and injuries related to screw drilling and placement. To decrease vascular complications to the popliteal artery during proximal tibial osteotomy, different knee positions have been recommended [5, 14, $29,31,40]$, based on the translation of the vessels during flexion and extension. In our study, all osteotomies were performed with the knee in extension to reproduce surgical approaches used at our institution. In addition, we used a 3-D reconstruction model rather than preestablished points on a two-dimensional image.

Characterizing the vascular pattern around the knee from the perspective of femoral and tibial osteotomies is important when considering potential complications. Dissection of the cadaver specimens provided graphic visual information, gave a comprehensive overview of the vessels at risk during surgery, and showed the potential of the extraosseous blood supply related to these osteotomies. Variations in branching patterns of the popliteal artery are not uncommon (10\%-15\% of cases) and several variations have been reported, the most common being a high 
division, and hypoplastic or aplastic posterior tibial artery $[1,6,11-13,15,17-20,22,25,29,30,41]$, but their implication in osteotomies around the knee rarely has been described $[15,28]$. To the best of our knowledge, the variant observed in our study (Fig. 4) has thus far been reported in only one knee [20]. The surgeon should be aware of these variations and their prevalence to limit the risk of injury while performing the osteotomy. It also was interesting to note the lack of bony vascularization on the subcutaneous medial side of the knee. This warrants further investigation on the possible affect it may have on bony healing in this area.

The major blood vessels around the knee were found to be at risk of injury during opening- and closing-wedge distal femoral and proximal tibial osteotomies. Mediumsized vessels and their tributaries were at risk around the osteotomy cuts but with the numbers available in our study, this vascular risk was not found to vary on the medial and lateral sides of the knee. The genicular arteries on the opposite side of the surgical field are at risk of injury during distal femoral and proximal tibial osteotomies as they cannot be seen in the approach and thus cannot be protected. More studies with larger sample sizes are needed to address the potential risk added by additional dissection for plate placement and that related to screw drilling and placement during osteotomies around the knee.

\section{References}

1. Bardsley JL, Staple TW. Variations in branching of the popliteal artery. Radiology. 1970;94:581-587.

2. Bonasia DE, Amendola A. Combined medial meniscal transplantation and high tibial osteotomy. Knee Surg Sports Traumatol Arthrosc. 2010;18:870-873.

3. Chae DJ, Shetty GM, Wang KH, Montalban AS Jr, Kim JI, Nha KW. Early complications of medial opening wedge high tibial osteotomy using autologous tricortical iliac bone graft and Tplate fixation. Knee. 2011;18:278-284.

4. Chahal J, Gross AE, Gross C, Mall N, Dwyer T, Chahal A, Whelan DB, Cole BJ. Outcomes of osteochondral allograft transplantation in the knee. Arthroscopy. 2013;29:575-588.

5. Coventry MB. Osteotomy about the knee for degenerative and rheumatoid arthritis. J Bone Joint Surg Am. 1973;55:23-48.

6. Day CP, Orme R. Popliteal artery branching patterns: an angiographic study. Clin Radiol. 2006;61:696-699.

7. Eriksson K, Bartlett J. Popliteal artery-tibial plateau relationship before and after total knee replacement: a prospective ultrasound study. Knee Surg Sports Traumatol Arthrosc. 2010;18:967-970.

8. Friel NA, Chu CR. The role of ACL injury in the development of posttraumatic knee osteoarthritis. Clin Sports Med. 2013;32:1-12.

9. Georgoulis AD, Makris CA, Papageorgiou CD, Moebius UG, Xenakis T, Soucacos PN. Nerve and vessel injuries during high tibial osteotomy combined with distal fibular osteotomy: a clinically relevant anatomic study. Knee Surg Sports Traumatol Arthrosc. 1999; 7:15-19.

10. Getgood A, Collins B, Slynarski K, Kurowska E, Parker D, Engebretsen L, MacDonald PB, Litchfield R. Short-term safety and efficacy of a novel high tibial osteotomy system: a case controlled study. Knee Surg Sports Traumatol Arthrosc. 2013;21:260-269.

11. Keen JA. A study of the arterial variations in the limbs with special reference to symmetry of vascular patterns. Am J Anat. 1961;108:245-261.

12. Kil SW, Jung GS. Anatomical variations of the popliteal artery and its tibial branches: analysis in 1242 extremities. Cardiovasc Intervent Radiol. 2009;32:233-240.

13. Kim D, Orron DE, Skillman JJ. Surgical significance of popliteal arterial variants: a unified angiographic classification. Ann Surg. 1989;210:776-781.

14. Kim J, Allaire R, Harner CD. Vascular safety during high tibial osteotomy: a cadaveric angiographic study. Am J Sports Med. 2010;38:810-815.

15. Klecker RJ, Winalski CS, Aliabadi P, Minas T. The aberrant anterior tibial artery: magnetic resonance appearance, prevalence, and surgical implications. Am J Sports Med. 2008;36:720-727.

16. Lee YS, Lee BK, Kim WS, Choi JS, Baek JR, Moon CW. Sagittal and coronal plane location of the popliteal artery in the openwedge high tibial osteotomy. Knee Surg Sports Traumatol Arthrosc. 2013 Apr 17. [Epub ahead of print]

17. Lippert H, Pabst R. Arterial Variations in Man: Classification and Frequency. Munich, Germany: JF Bergman Verlag; 1985.

18. Mauro MA, Jaques PF, Moore M. The popliteal artery and its branches: embryologic basis of normal and variant anatomy. AJR Am J Roentgenol. 1988;150:435-437.

19. Morris GC Jr, Beall AC Jr, Berry WB, Feste J, De Bakey ME. Anatomical studies of the distal popliteal artery and its branches. Surg Forum. 1960;10:498-502.

20. Ozgur Z, Ucerler H, Aktan Ikiz ZA. Branching patterns of the popliteal artery and its clinical importance. Surg Radiol Anat. 2009;31:357-362.

21. Phisitkul P, Haugsdal J, Vaseenon T, Pizzimenti MA. Vascular disruption of the talus: comparison of two approaches for triple arthrodesis. Foot Ankle Int. 2013;34:568-574.

22. Piral T, Germain M, Princ G. Absence of the posterior tibial artery: implications for free transplants of the fibula. Surg Radiol Anat. 1996;18:155-158.

23. Roos H, Laurén M, Adalberth T, Roos EM, Jonsson K, Lohmander LS. Knee osteoarthritis after meniscectomy: prevalence of radiographic changes after twenty-one years, compared with matched controls. Arthritis Rheum. 1998;41:687-693.

24. Sabalbal M, Johnson M, McAlister V. Absence of the genicular arterial anastomosis as generally depicted in textbooks. Ann $R$ Coll Surg Engl. 2013;95:405-409.

25. Sanders RJ, Alston GK. Variations and anomalies of the popliteal and tibial arteries. Am J Surg. 1986;152:531-534.

26. Savarese E, Bisicchia S, Romeo R, Amendola A. Role of high tibial osteotomy in chronic injuries of posterior cruciate ligament and posterolateral corner. J Orthop Traumatol. 2011;12:1-17.

27. Sawant MR, Ireland J. Pseudo-aneurysm of the anterior tibial artery complicating high tibial osteotomy: a case report. Knee. 2001;8:247-248.

28. Shenoy PM, Oh HK, Choi JY, Yoo SH, Han SB, Yoon JR, Koo JS, Nha KW. Pseudoaneurysm of the popliteal artery complicating medial opening wedge high tibial osteotomy. Orthopedics. 2009;32:442.

29. Shetty AA, Tindall AJ, Qureshi F, Divekar M, Fernando KW. The effect of knee flexion on the popliteal artery and its surgical significance. J Bone Joint Surg Br. 2003;85:218-222.

30. Singla R, Kaushal S, Chabbra U. Popliteal artery branching pattern: a cadaveric study. Eur J Anat. 2012;16:157-162.

31. Smith PN, Gelinas J, Kennedy K, Thain L, Rorabeck CH, Bourne RB. Popliteal vessels in knee surgery: a magnetic resonance imaging study. Clin Orthop Relat Res. 1999;367:158-164. 
32. Smith TO, Sexton D, Mitchell P, Hing CB. Opening- or closingwedged high tibial osteotomy: a meta-analysis of clinical and radiological outcomes. Knee. 2011;18:361-368.

33. Szyber P Jr, Skóra J, Rybak W, Pupka A. Iatrogenic pseudoaneurysm of the popliteal artery following corrective tibial osteotomy. Vasa. 2011;40:414-417.

34. Tennant JN, Rungprai C, Pizzimenti MA, Goetz J, Phisitkul P, Femino J, Amendola A. Risks to the blood supply of the talus with four methods of total ankle arthroplasty: a cadaveric injection study. J Bone Joint Surg Am. 2014;96:395-402.

35. Vena G, D'Adamio S, Amendola A. Complications of osteotomies about the knee. Sports Med Arthrosc. 2013;21:113-120.

36. Virolainen P, Aro HT. High tibial osteotomy for the treatment of osteoarthritis of the knee: a review of the literature and a metaanalysis of follow-up studies. Arch Orthop Trauma Surg. 2004;124:258-261.
37. Visser J, Brinkman JM, Bleys RL, Castelein RM, van Heerwaarden RJ. The safety and feasibility of a less invasive distal femur closing wedge osteotomy technique: a cadaveric dissection study of the medial aspect of the distal femur. Knee Surg Sports Traumatol Arthrosc. 2013;21:220-227.

38. Wang JW, Hsu CC. Distal femoral varus osteotomy for osteoarthritis of the knee. J Bone Joint Surg Am. 2005;87: 127-133.

39. Willey M, Wolf BR, Kocaglu B, Amendola A. Complications associated with realignment osteotomy of the knee performed simultaneously with additional reconstructive procedures. Iowa Orthop J. 2010;30:55-60.

40. Zaidi SH, Cobb AG, Bentley G. Danger to the popliteal artery in high tibial osteotomy. J Bone Joint Surg Br. 1995;77:384-386.

41. Zwass A, Abdelwahab IF. A case report of anomalous branching of the popliteal artery. Angiology. 1986;37:132-135. 